\title{
Intervenciones de trabajadores y trabajadoras sociales en contextos de violencia de género
}

Interventions of social workers in contexts of gender violence

Cory Duarte Hidalgo

Doctora en Trabajo Social

(Universidad Complutense de Madrid)

Profesora asociada en el Departamento de

Trabajo Social

(Universidad de Atacama)

Correo: cory.duarte@uda.cl

Viviana Rodríguez Venegas

Doctoranda en Estudios Sociales de América

Latina

(Universidad Nacional de Córdoba)

Profesora asociada en el Departamento de

Trabajo Social

(Universidad de Atacama)

Correo: viviana.rodriguez@uda.cl
Anais Araya Rojas

Licenciada en Trabajo Social

(Universidad de Atacama)

Correo: anais.araya.16@alumnos.uda.cl

Giannina Maya Loayza

Licenciada en Trabajo Social

(Universidad de Atacama)

Correo: mayazf@hotmail.com

Maricela Ulloa Muñoz

Licenciada en Trabajo Social

(Universidad de Atacama)

Correo: maricela.ulloa.munoz@outlook.com 


\section{Resumen}

La investigación se basa en una revisión bibliográfica que toma como corpus una serie de artículos publicados en distintas revistas iberoamericanas sobre intervenciones realizadas por trabajadores y trabajadoras sociales en contextos de violencia de género. El estudio, de tipo bibliográfico, analizó los temas tratados, las estrategias interventivas y las reflexiones que, desde el Trabajo Social, se realizan al respecto. Los principales resultados señalan la utilización de la consideración de la violencia de género como una vulneración de derechos, el uso de la perspectiva de género en la intervención, sin embargo, su uso presenta dificultades en el abordaje práctico debido a la escasa formación en la temática y la influencia de otros factores relevantes de considerar en este tipo de procesos de intervención, además de identificar elementos metodológicos y éticos en el abordaje de este tipo de situaciones. Asimismo, se genera una discusión respecto de los desafíos y dificultades que presenta el contexto de crisis socio-sanitaria en la región, estableciendo la necesidad de repensar las prácticas y estrategias interventivas desarrolladas en contextos de violencia de género.

Palabras clave

Trabajo Social, Intervención Social, Violencia de Género, Perspectiva de Género

\section{Abstract}

The research is based on a bibliographic review that takes as a corpus a series of articles published in different Ibero-American journals on interventions carried out by social workers in contexts of gender violence. The study, of a bibliographic type, analyzed the topics discussed, the intervention strategies and the reflections that, from the Social Work, are carried out in this regard. The main results indicate the use of the consideration of gender violence as a violation of rights, the use of the gender perspective in the intervention, however, its use presents difficulties in the practical approach due to the scarce training in the subject and the influence of other relevant factors to consider in this type of intervention process, in addition to identifying methodological and ethical elements in the approach to this type of situation. Likewise, a discussion is generated regarding the challenges and difficulties presented by the context of the socio-sanitary crisis in the region, establishing the need to rethink the interventional practices and strategies developed in contexts of gender violence.

Keywords

Keywords: Social Work, Social Intervention, Gender Violence, Gender Perspective 


\section{Introducción ${ }^{1}$}

En trabajos anteriores hemos señalado la necesidad de incorporar la perspectiva de género en los debates contemporáneos del trabajo social, considerando esto como un asunto ineludible, pero sobre el cual existe una serie de ausencias y temas no visibilizados (Duarte y Rodríguez, 2020) que son necesarios de enfrentar y considerar para avanzar en una necesaria despatriarcalización de nuestra profesión (Duarte, 2021). No obstante, y antes de avanzar en la presentación del trabajo realizado, conviene problematizar el constructo género como una noción que alude a una "perspectiva pluriversa de análisis, activismo y transformación”, un "conjunto díscolo de prácticas sentipensantes y activistas de construcción de un mundo otro" (Roldán, Failla y Hermida, 2019:1), en permanente tensión contra las lógicas heteropatriarcales, coloniales y capitalistas. Desde esta perspectiva, resultaba necesario para quienes investigan, generar una indagación sobre lo escrito respecto de la consideración de la perspectiva de género en el marco de las intervenciones que se realizan en la profesión, con la finalidad de discutir, desde un posicionamiento situado y crítico, las construcciones en la relación/tensión entre intervención social y género.

La intervención social, entendida como "un proceso epistemológica y políticamente construido, planificado para la consecución de un cambio significado como deseable, implementado a través de estrategias, métodos y técnicas específicas y (en el mejor de los casos) evaluado y retroalimentado" (Muñoz, 2014:38), es una forma de mirar, una perspectiva que nos permite situarnos desde un ámbito multidimensional y poliédrico en el que se interrelacionan aspectos políticos, éticos, teóricos y metodológicos, asociados a la transformación social. La intervención social es siempre una construcción de carácter sociopolítico (Muñoz, 2014), que se articula en función de nuevas formas de subjetividades (Castro y Gutiérrez, 2017), marcadas por la subalternidad, la opresión, la violencia y la vulneración de derechos (Hermida,

$1 \mathrm{El}$ presente artículo forma parte de la investigación "Memorias colectivas y procesos de resistencia comunitaria de las mujeres en Atacama" financiado con fondos de la Vicerrectoría de Investigación y Posgrado de la Universidad de Atacama. 
2017), en un escenario complejo en el cual, el acompañamiento social (Zamanillo, 2004) se realiza en medio de una tensión constante entre las demandas ciudadanas y las escasas respuestas entregadas por la institucionalidad (Meschini y Rampoldi, 2017). A estos asuntos se suman los condicionantes institucionales, las características de los sujetos, la formación académica y fines propios de cada profesional (Falla, 2017), aspectos que se transforman en un desafío constante para los y las profesionales, en contextos contradictorios en los que deben enfrentar una serie de dilemas y problemas éticos derivados de la acción profesional.

En la misma línea, es relevante ahondar respecto de los procesos interventivos desarrollados en contextos de violencia de género, pues, tal como señala Fernández (2015), existe una alta presencia de profesionales de Trabajo Social en esta temática, en una profesión altamente feminizada (Lorente, 2002; Duarte, 2013), en la que existen mujeres interviniendo con mujeres (Alcázar, 2012; Fernández, 2015), relación en la que profesionales y personas en intervención se encuentran insertas en un sistema de opresión y una estructura social y política, influenciada por una cultura machista y patriarcal e intersectadas por múltiples ejes. En relación con lo descrito, el equipo de trabajo considera importante analizar y comprender estos procesos interventivos, ya que actualmente la labor que realizan los y las profesionales de Trabajo Social en contextos de violencia no es transmitida, sistematizada y/o teorizada, sumado a que su desempeño no solo se enfoca en una única tarea en la intervención directa, sino que es amplia y diversa.

\section{Metodología}

La investigación se articuló como una revisión bibliográfica, en la que se aplicaron criterios de inclusión de tipo temporal y palabras claves. De esta forma, el corpus fue integrado por un total de 84 artículos preseleccionados los que pasaron a un análisis más exhaustivo y detallado para determinar su pertinencia con la investigación. 
Tabla 1: Criterios de inclusión y exclusión

\begin{tabular}{|l|l|}
\hline Tipo de enfoque & Cualitativo o mixto \\
\hline Conceptos claves & $\begin{array}{l}\text { Considera en el título o en la selección de con- } \\
\text { ceptos claves, al menos una de las siguientes } \\
\text { palabras: intervención social, Trabajo Social, } \\
\text { violencia de género, mujeres }\end{array}$ \\
\hline Criterio temporal & Estudios publicados entre el año 2004-20201 \\
\hline Criterio lingüístico & Estudios publicados en idioma español \\
\hline Criterio de exclusión & $\begin{array}{l}\text { Artículos catalogados como: proyectos de } \\
\text { tesis o tesis, artículos grises, artículos de opi- } \\
\text { nión o reflexión, entrevistas }\end{array}$ \\
\hline
\end{tabular}

Fuente: Elaboración propia.

Los artículos fueron registrados en una planilla, para luego efectuar una evaluación de los datos, lo que llevó a una selección final de treinta artículos. Posteriormente, se realizó una lectura comprensiva acompañada de un proceso sistemático para analizar de manera exhaustiva cada uno de aquellos, entregando una mayor rigurosidad y transparencia al trabajo elaborado. Estas decisiones metodológicas permitieron que el proceso de organización y tratamiento de datos estuviese en una constante revisión para la verificación de su coherencia y veracidad. Respecto del tipo de análisis de datos cualitativos utilizado, se realizó mediante el análisis de contenido, siguiendo los pasos planteados por Guix (2007), el que fue asistido mediante el uso del programa computacional Atlas. Ti versión 8.4

\section{Resultados}

En el material analizado, se puede observar que la intervención del Trabajo Social en esta temática, reconoce la violencia de género como una vulneración a los Derechos Humanos2 (Piedra et al., 2018),

2 Si bien es cierto, el Derecho Internacional de los Derechos Humanos (DIDH) solo hace referencia a la violencia contra las mujeres, evadiendo la categoría género, si queda claro, en la mayoría de los artículos, que la discriminación y la violencia contra las mujeres es una violación a los Derechos Humanos. En este sentido, puede entenderse por 
enraizada en la discriminación que han sufrido históricamente las mujeres y cuerpos feminizados, en el marco de una violencia de carácter estructural que pretende "perpetuar el sistema de jerarquías que nos ha impuesto el patriarcado" (Cánovas et al., 2017). De esta forma, la violencia de género es considerada como la "máxima expresión de la desigualdad entre varones y todas las identidades oprimidas (cis-mujeres, y el colectivo LGBTIQ)" (Sánchez y Percara, 2017:12) asegurando, a través de la violencia, la instauración de elementos asociados a la legitimización del heteropatriarcado como sistema de dominación y opresión.

Conceptualizar este tipo de violencias como de género permite resignificar la violencia contra la mujer, ya que esta se basa en el "sostenimiento estructural y relacional de roles que no son naturales sino construidos, aprendidos culturalmente, desiguales e injustos" (Campos et al., 2018:32). Así, la violencia deja de ser un problema privado, situado únicamente entre el agresor y la víctima, para ser comprendido como una violencia estructural que afecta a la sociedad en su conjunto. La violencia de género se asume como un problema público, en el que los Estados deben tomar cartas en el asunto e intervenir en todos los ámbitos y contextos donde ésta se presente (Clariá, Condori y Rivolta, 2018), dando cumplimiento a los compromisos internacionales adquiridos en función de la protección y garantía de derechos de todas las personas. No obstante, la disminución de los servicios sociales y la escasa atención e intervención de la violencia de género, así como lo limitado de los recursos y dispositivos diseñados para estos fines, dificulta la atención integral a las víctimas, contribuyendo al aumento de los índices de violencia (Cotalero, 2015).

La violencia de género presenta y adopta distintas modalidades (Piedra et al., 2018), en distintos ámbitos (Campos et al, 2018) y con-

violencia de género en el marco del DIDH "aquella cometida contra hombres o mujeres, con fundamento en conceptos normativos expresados en instituciones y construidos sobre los símbolos disponibles de lo masculino y lo femenino en un plano jerárquico y discriminatorio, que se traduce en injusticias sobre el reconocimiento identitario de las personas, la distribución de cargas y beneficios, y el control propio de la vida sexual o de otras opciones personales, por el hecho mismo de representarse como hombres o mujeres" (Orjuela, 2012:110). 
textos sociales (Cotalero, 2015), expresándose de "formas muy variadas que van desde lo público a lo privado, de lo físico a lo psicológico, pasando por formas más sutil y más difíciles de medir como la discriminación laboral y el 'techo de cristal' o el acoso callejero" (Cánovas et al, 2017:31). A pesar de este reconocimiento sobre la diversidad de formas y contextos en los que se desarrolla la violencia de género, se realiza un mayor número de investigaciones e intervenciones en aquellas situadas en contextos familiares o en relaciones de pareja (Bravo, 2008; Villaluenga, 2020). Este aspecto es significativo debido a que se tiende a poner énfasis en asuntos como la afectación de la violencia en el ejercicio de maternajes (Plaza y Cantera, 2016), por sobre la situación específica que viven las mujeres y disidencias sexo-genéricas como víctimas de violencia. Por otra parte, Danis y Lockart (2003, citado en Santana 2010), mencionan que desde finales de la década de los setenta y hasta principios de los noventa del siglo pasado, la profesión fue criticada por mantener un trato indiferente y desinformado respecto a las mujeres maltratadas. En este sentido, algunos estudios revelaron que las trabajadoras y los trabajadores sociales cometen el error de culpar a la víctima (Davis y Carlson, 1981, citado en Santana, 2010) y mantener una actitud enmarcada en los prejuicios y connotaciones sexistas a la hora de intervenir.

El quehacer profesional de los trabajadores y trabajadoras sociales en contexto de violencia de género se enmarca en un conjunto de orientaciones predefinidas por las instituciones que habitan, sin embargo, cada profesional toma decisiones, asociadas a las propias definiciones y posicionamientos previos sobre la temática, lo que marcará la intervención desarrollada y la relación que tengan con las personas que viven alguna situación de violencia (Campos, Commisso, Núñez y Díaz, 2018). La inclusión de la perspectiva de género en los procesos interventivos permite incorporar un conjunto de estrategias y herramientas orientadas hacia una mirada reflexiva de la realidad y con ello, el camino para una transformación social (Chacón, 2019). En este sentido, es preciso destacar que, a pesar de que en la actualidad la acción profesional tiene un sustento sólido en la perspectiva de derechos, la incorporación de la perspectiva de género sigue siendo un 
desafío pendiente para enfrentar integralmente la violencia (Campos et al., 2018).

La intervención con personas que han sufrido violencia de género implica trabajar elementos que cuestionan lo establecido por la razón patriarcal (Nogueiras, Blanc y Pliego, 2005), lo cual exige romper con patrones culturales respaldados por un sistema androcentrista y misógino que afectan las concepciones tradicionales de la intervención social. De esta forma, se debe comprender que la inclusión de la perspectiva de género en estos procesos puede superar los propios límites de la relación interventiva, por lo que se hace necesario considerar miradas críticas que logren problematizar y cuestionar estos aspectos, relevando aspectos éticos como el respeto a la autonomía, el resguardo de la privacidad y confidencialidad (Aveiga y Ponce, 2019). Asimismo, es importante para el desarrollo de una intervención efectiva, la implementación de estrategias apropiadas que consideren los elementos contextuales y otros asociados al tipo de violencia y su grado de afectación (Campos et al., 2018), siendo clave la relación profesional que se establece con la persona que ha vivenciado violencia de género (Villaluenga, 2020). En este sentido, la intervención en estos contextos requiere no solo comprensión de las bases teóricas y conceptuales, sino también conocer los fundamentos sociales y culturales que perpetúan la violencia (Ullastre, 2018), así como sus particularidades en el caso de las diversidades y disidencias sexo-genéricas.

Una de las grandes críticas encontradas en la revisión bibliográfica alude a que las propuestas formativas en Trabajo Social no logran entregar herramientas metodológicas y teóricas sobre violencia de género. La escasez de profesionales con formación en enfoque de derechos y perspectiva de género ocasiona dificultades a la hora de garantizar la atención integral, adecuada y pertinente a las personas que vivencian algún tipo de violencia (Chacón, 2019). En este sentido, se hace necesario incorporar en las universidades espacios de conocimiento, discusión y reflexión que permitan introducir la perspectiva de género en los planes de estudio (Santana, 2010), formando profesionales capaces de detectar e intervenir en contextos de violencia de género (Elboj y Ruíz, 2010). 
En términos metodológicos, los artículos revisados revelan la importancia de la entrevista inicial o entrevista de acogida, la cual es descrita como el momento clave para crear un ambiente de confianza, recoger antecedentes y entregar la orientación necesaria (Álava, Alcívar y Macias, 2019). Este primer momento es considerado por Campos et al., (2018) como un "encuentro dialógico" en el que interactúan distintas posiciones de poder, teniendo en claro que la "cultura patriarcal nos atraviesa, marcando el contenido de los relatos, y desplegándose a través de prejuicios que pueden obstaculizar la escucha" (2018:39). En la misma línea, Canovas, Gallego, Navarro y González (2017) refieren que, durante la entrevista, es fundamental evitar los prejuicios, conservar una actitud positiva y mantener especial atención en la comunicación no verbal. En este encuentro inicial se acuerda el proceso interventivo, sus alcances, estrategias, etapas propuestas y relevancia de la intervención social, considerando esta como un proceso de acuerdo entre el/la profesional y la persona afectada (Clariá et al., 2018). Por ello es relevante destacar la importancia y valor del consentimiento de la persona durante todas las etapas del proceso interventivo (Clariá et al., 2018; Álava et al., 2019), entregando con ello, toda la información necesaria para que la persona afectada pueda tomar decisiones por sí misma, fomentando la autonomía y "recuperación global que precisa tras la destrucción que acarrea la violencia" (Villaluenga, 2020:84). En este sentido, es importante situar a la persona no desde su reconocimiento como víctima, lo que genera "propuestas de control y normativización sobre sus cuerpos y sus formas de nominación” (Galaz y Guarderas, 2017:80), sino más bien, considerarle como una persona "que se encuentra en una situación de violencia género, lo cual permite pensar que esa situación es transitoria y se puede terminar" (Sánchez y Percara, 2017:15), visualizándoles como sujetas activas de derecho y protagonistas de sus procesos (Clariá et al., 2018).

Otro aspecto relevante a destacar es que la implementación de acciones preventivas se ven dificultadas por lógicas gerencialistas que evalúan la intervención profesional a través de la medición de sus impactos directos (Romero, 2019). A pesar de lo anterior, se destaca la necesidad de generar estrategias de prevención que propicien instancias de diálogo y reflexión en torno a prácticas y patrones naturaliza- 
das de violencia patriarcal (Cánovas et al., 2017), apuntando hacia su disminución (Álava et al., 2019), materia en que el Trabajo Social resulta ser clave (Ríos 2003, Álava y Veliz, 2018). Asimismo, es necesario generar un trabajo interdisciplinario e integral (Villaluenga, 2020), que potencie espacios seguros (Amoroso y Fernández, 2014; Ullastre, 2018) y estratégicos para la detección de la violencia, la intervención y la articulación de acciones (Amoroso y Fernández, 2014).

Lo expuesto, invita a reflexionar sobre los aspectos que involucran la relación interpersonal y profesional con las personas que han vivenciado violencia de género, así también es necesario considerar el posicionamiento ético-político de los trabajadores y trabajadoras sociales, evaluando si favorecen el cambio estructural de las instituciones o acatan pasivamente lo impuesto (Campos et al., 2018). De esta forma, se hace urgente politizar la práctica: politizar en sentido de develar, explicitar, descubrir, las contradicciones de intereses, las tensiones (manifiestas o latentes, explícitas o implícitas) existentes entre los y las sujetas y actoras de las realidades donde interviene el profesional y explicitar las diferencias de intereses entre la institución y las sujetas que transitan el dispositivo (Clariá et al., 2018).

En este sentido, resulta relevante repensar los dilemas y problemas éticos que transitan los trabajadores y trabajadoras sociales desde una perspectiva de género, poniendo atención sobre las relaciones entre ética y género en Trabajo Social.

La profesión de Trabajo Social se realiza en una posición situada, encarnada y subjetiva también de género, con la que construimos el conocimiento para nuestra intervención. El desafío ético, tanto teórico como metodológico, pasa por incorporar las emociones y las contradicciones que tenemos en nuestro ejercicio profesional que, en aras de la supuesta objetividad y cientificidad del Trabajo Social, tradicionalmente han sido reprimidas y silenciadas (Agrela, Gutiérrez y Fernández, 2017:62).

Diversos autores y autoras concuerdan en que una de las falencias en la intervención de trabajadores y trabajadoras sociales en violencia de género es la fundamentación teórica (Amoroso y Fernández, 2014; Tudela, 2015; Campos et al., 2018; Chacón, 2019; Romero, 2019; Villaluenga, 2020), lo cual estaría relacionado con instituciones educati- 
vas e interventivas marcadas por lógicas patriarcales (Romero, 2019), que dejan la formación y capacitación en perspectiva de género como un elemento voluntario u opcional (Amoroso y Fernández, 2014). En la información recabada de la revisión de artículos, destaca la perspectiva de género como el enfoque más influyente y utilizado (Santana, 2010, Piedra et al., 2018, Chacón 2019; Romero, 2019), apropiado para el abordaje de estos asuntos, aunque se logra identificar la utilización de otros enfoques o perspectivas en la intervención en la materia.

La perspectiva de género "implica una posición política frente a la opresión de género, que busca denunciar los daños provocados por ésta y, al mismo tiempo, ser un conjunto de acciones y alternativas para erradicar tal opresión" (Chacón, 2019:84). La incorporación de la perspectiva de género permitiría situarse desde la promoción y autonomía de las mujeres, bajo el fundamento de la defensa de sus derechos e igualdad de oportunidades (Santana, 2010); al mismo tiempo, facilita la toma de conciencia sobre las relaciones de poder en los procesos de intervención social y la identificación de las prácticas y discursos que reproducen y perpetúan la desigualdad (Romero, 2019). Así también, la implementación de intervenciones enfocadas desde esta perspectiva favorecería el desarrollo de acciones apropiadas para cada necesidad y realidad (Tudela, 2015), siendo fundamental que los y las profesionales tengan una conceptualización clara, lo que permitiría problematizar las intervenciones que se enmarcan en lógicas de dominación y control patriarcal tanto en las relaciones y acciones cotidianas de trabajadores y trabajadoras sociales como en las instituciones ejecutoras (Campos et al., 2018). En esta misma dirección es necesario reconocer la responsabilidad que como gremio tenemos en la perpetuación de las inequidades sexo-genéricas (Fernández, 2015; Romero, 2019), debido a que los y las profesionales no escapan del patriarcado y a que los contextos institucionales marcan la actuación profesional, replicando las estructuras de poder dominantes. Esta situación genera que los y las profesionales realicen su trabajo en un sistema que no comprende la complejidad de la violencia de género, anquilosados en modalidades ya impuestas que no suelen transformar o cambiar (Romero, 2019). Ante este escenario, se considera relevante promover una actuación profesional antipatriarcal (Duarte, 2021) que 
logre incomodar las instituciones y contextos que habitan, pues "no se puede pensar la violencia de género como problema social y público, desde instituciones y justicia patriarcales" (Clariá et al., 2018:19). La declaración política de un trabajo social antipatriarcal parte del reconocimiento de las propias contradicciones, colocándolas en discusión, permitiéndoles estar pendientes de aquellos elementos que pueden obstaculizar la intervención (Campos et al., 2018).

En la revisión bibliográfica se observa una marcada presencia de estrategias derivadas de las teorías feminista en la intervención en violencia de género, sobre todo en las publicaciones más recientes. Elementos como la consideración del sistema de relaciones de género (De la Fuente, 2012) y la interseccionalidad (Chacón, 2019; Tudela, 2015, Duarte y Rodríguez, 2020), permite cuestionar la idea que la violencia afecta a todas por igual, incorporando elementos raciales, de clase social, religión, edad, territorio y otros, en la comprensión de la temática.

Cabe señalar que en el material observado, se distinguen otras aproximaciones como la amplia utilización de la perspectiva ecológica, para analizar la forma en que los diversos factores societales inciden en la causa, reproducción y transformación de la violencia. A esto se suma la utilización de la teoría del ciclo de la violencia en contextos familiares y de pareja (Piedra et al., 2018). A su vez, Ríos (2003) expone diferencias en los enfoques que se utilizan en la intervención, marcados por dos extremos, uno de carácter asistencial-funcionalista y otro de tipo progresista (2010), sin embargo, prevalecen dispositivos orientados al control social, por encima de acciones que les permitan comprender las estructuras de violencia y la entrega de recursos para enfrentarlas, no solo enfocados en el ámbito jurídico, sino más bien en la consideración de una intervención integral que incorpore modificaciones en los ámbitos sociales y económicos de las personas afectadas (2014).

\section{Discusión}

La consideración de la perspectiva de género en Trabajo Social es un tema que ha tomado mayor fuerza a partir de los últimos años, principalmente por la actuación del movimiento feminista, el cual ha 
logrado introducir debates y cuestionamientos en las formas de intervención e investigación. Desde la academia se observa una mayor reflexión sobre estos temas, sin embargo, tal y como se evidencia en la información recolectada, queda un gran camino por transitar, puesto que aún se observa una "depreciación en la difusión de los temas relacionados con conocimientos aplicados para la intervención social desde/con/para las mujeres" (Agrela y Morales, 2018:2). Sin embargo, no podemos considerar la perspectiva de género enfocada solo en mujeres, sino más bien, debe reconocer "la existencia de diversos géneros y sus relaciones de poder, que se imbrican con otros elementos generadores de desigualdad, tales como la etnia/raza, la clase social, el origen nacional" (Alcazar, 2012:100). Este elemento resulta particularmente importante, pues la consideración de la perspectiva está asociada al cuestionamiento y enfrentamiento de las relaciones de poder entre los géneros, considerando a su vez las diversidades y las disidencias al ordenamiento genérico tradicional. En este sentido, y como lo vemos en los resultados, resulta urgente incorporar la perspectiva de género en la formación de trabajadores y trabajadoras sociales, con tal de garantizar intervenciones adecuadas y pertinentes en estas y otras materias (Méndez y Álvarez-Buylla, 2007; Penyarroja, 2021), de lo contrario "las mujeres corren el riesgo de ser atendidas por equipos que no están capacitados para intervenir en estas situaciones y en los que las mujeres son percibidas como víctimas de problemas individuales y psicológicos" (Penyarroja, 2021:37). Estos elementos resultan claves en los procesos de intervención, sobre todo en la consideración y respeto de la autonomía de las mujeres y disidencias, quienes pueden generar procesos de concienciación sobre los procesos vividos, permitiéndole reconocerse no solo en situaciones de violencia con sujetos particulares, sino como una persona que ha desarrollado su vida en una sociedad patriarcal que establece la violencia como medio de relación (Bosch, Ferrer y Alzamora, 2005).

A su vez, es necesario problematizar las intervenciones que se realizan desde miradas patriarcales centradas en lo socio-laboral, o en la reparación del trauma, o aumento de la autoestima (Penyarroja, 2021). En este sentido, como equipo, apostamos por intervenciones feministas que pongan el foco en develar las estructuras elementales de 
la violencia (Segato, 2010), que generen procesos de toma de conciencia y emancipación, priorizando el acompañamiento, dejando de lado la rigidez de los protocolos (Penyarroja, 2021), y que puedan apostar por los protagonismos y autonomía de las mujeres, niñas y disidencias.

No obstante, es necesario problematizar y visibilizar las condiciones en las que se desempeñan los y las profesionales que intervienen en estas materias, pues, en muchos casos, su desempeño laboral está marcado por la precariedad e inseguridad laboral, en programas e instituciones que presentan una mirada patriarcal que no reconoce ni valora el trabajo realizado, dando por hecho "que las profesionales que trabajan en este ámbito recibirán una parte de su retribución en forma de salario afectivo y que, educadas para el cuidado, las profesionales asumirán de forma natural esta cláusula contractual no escrita" (Méndez y Álvarez-Buylla, 2007:293)

Las restricciones derivadas de la crisis socio-sanitaria generada por la pandemia de Covid-19 han aumentado la violencia de género, potenciando, a través del confinamiento, las situaciones y condiciones de riesgo en que se encuentran quienes viven situaciones de violencia (Montenegro y Montenegro, 2020; Lorente, 2020; 2021), suponiendo una especie de "doble confinamiento para las mujeres" (Mateos, 2021:108), que no es expresado necesariamente en las estadísticas sobre violencia (Lorente, 2020). El confinamiento ha generado una disminución en las denuncias formales (Londoño, 2020; Carrasco y Martínez, 2020; Ruiz y Pastor, 2021), debido principalmente a las restricciones de desplazamiento (Izquierdo, 2020), pero al mismo tiempo, se observa un incremento en las solicitudes de ayuda y orientación a través de líneas telefónicas (Montenegro y Montenegro, 2020; Izquierdo, 2020; Carrasco y Martínez, 2020; Ruiz y Pastor, 2021), en la ocupación de los refugios (Montenegro y Montenegro, 2020) y el aumento de los femicidios (Carrasco y Martínez, 2020). Además, la pandemia ha evidenciado las falencias del sistema jurídico de protección, expresadas en profundas dificultades en el acceso a la justicia, así como el cierre de dispositivos y programas relacionados con protección de víctimas, y otros relacionados con derechos sexuales y reproductivos (Londoño, 2020), observándose serias dificultades en la detección y atención de casos de violencia (Penyarroja, 2021). Así 
mismo, se observa el aumento de la violencia patrimonial y económica en la medida que más mujeres están expuestas al desempleo, precarización y pobreza (Londoño, 2020). En este mismo orden coas, otro elemento preocupante es el aumento de la violencia sexual perpetrada contra mujeres y niñas, quienes viven confinamientos y cuarentenas junto a sus agresores (Londoño, 2020), lo que suma a las dificultades existentes para la articulación de redes de apoyo y soporte comunitario (Penyarroja, 2021).

Los elementos enunciados anteriormente, evidencian la necesidad de reforzar las intervenciones en estos escenarios, generando cambios y propuestas que permitan enfrentar el nuevo contexto mundial. En términos de la intervención social en violencia de género, se hace necesario evaluar, discutir y problematizar los indicadores e instrumentos usados, poniendo especial énfasis en las adaptaciones de los mismos en relación a poder pesquisar con mayor precisión los niveles de riesgo (Lorente, 2020) en los que se encuentran las mujeres y cuerpos feminizados durante la pandemia y posterior a la misma.

Estos nuevos escenarios hacen cuestionar las formas en que como profesión enfrentamos la violencia de género, pues, su abordaje, a la luz de lo revisado en el artículo, ocupa un lugar marginal en la formación y la intervención del trabajo social, recurriendo, en varias ocasiones a marcos teóricos y metodológicos que no han tenido mayor modificación en las últimas décadas, reproduciendo orientaciones técnicas institucionales que trasladan fórmulas de una intervención a otra sin mayor detención ni adecuación. A nuestro parecer, se requiere reforzar los conocimientos y estrategias desplegados, con una perspectiva de género crítica, que permita generar innovaciones y acciones que signifiquen alternativas de protección real de las mujeres, niñas y cuerpos feminizados ante la violencia de género. Con esto no responsabilizamos a la profesión de la mitigación de la violencia, sino más bien, afirmamos que esta se encuentra limitada en repertorios que no logran modificar las situaciones de violencia, precisamente, porque no cuentan con los andamiajes necesarios para ello. Sumado a ello, se debe considerar, tal y como aparecía en los resultados, las condiciones laborales que enfrentan los trabajadores y trabajadoras sociales, las que en muchos casos, se precarizaron y agudizaron durante la pan- 
demia (Muñoz, Reininger, Villalobos y Morales, 2021), generando dificultades ante la creciente demanda (Montenegro y Montenegro, 2020), por lo que se requieren equipos capacitados y formados en perspectiva de género, que cuenten con "elementos de trabajo adecuados, pago y condiciones laborales justas" (Carrasco y Martínez, 2020:52).

Creemos que la crisis socio-sanitaria es una oportunidad para repensar la praxis, generando reflexiones y análisis que permitan reorientar las estrategias y herramientas desplegadas en la intervención (Montenegro y Montenegro, 2020; Penyarroja, 2021), repensando las lógicas de acción (Carrasco y Martínez, 2020), permitiendo adecuaciones rápidas a las nuevas formas de cautiverio y violencia, generando adaptaciones a los contexto de crisis, evitando la atomización (Méndez y Álvarez-Buylla, 2007), y creando propuestas comunitarias que permitan subvertir las lógicas de un sistema que pareciera fortalecerse por medio de la pandemia (Quispe, 2020). En este sentido, es necesario incorporar el apoyo telefónico o virtual, así como las intervenciones en línea, teniendo siempre en consideración los aspectos diferenciales e interseccionales (Ford-Gilboe et al., 2020), no obstante, la implementación de estas estrategias requieren demandar a los Estados para proveer de los servicios de internet y equipos necesarios como una forma de entregar protección a las víctimas (Montenegro y Montenegro, 2020), así como el aumento de lugares de acogida y protección para las personas que han sufrido violencia (Ruiz y Pastor, 2021), lo que implica destinar recursos efectivos y no solo dar continuidad a las escuálidas acciones existentes desde antes de la pandemia.

Quisiéramos también señalar aquí la necesidad de generar estrategias de resistencia profesional (Strier y Breshtling, 2016) que permitan develar la racionalidad neoliberal imperante y que den cuenta de las opresiones que el sistema capitalista, colonial y heteropatriarcal realiza sobre los trabajadores y trabajadoras sociales, condicionado formas de intervención que puedan desplegar y articular oportunidades de transformación, y prácticas emancipatorias contra el orden hegemónico (Muñoz, 2020). En el contexto de la crisis socio-sanitaria que la que estamos inmersas, resulta más urgente que nunca el politizar la práctica, resistir y subvertir las lógicas hegemónicas, despa- 
triarcalizando el Trabajo Social, fortaleciendo las prácticas políticas de esperanza (Duarte, 2021), reconociéndonos como agentes de cambio y transformación, en articulación con las organizaciones locales y comunitarias, los movimientos sociales, los feminismos, las asociaciones profesionales, el colegio profesional (Muñoz, 2018) como estrategia viva de resistencia y acción.

\section{Referencias bibliográficas}

Álava, Leila María, Alcívar, Eva Margarita y Macías, Steeven Andrés (2019). Protocolos de intervención del trabajador social en casos de violencia intrafamiliar en la Fundación Nuevos Horizontes, Cantón Portoviejo, periodo 2019. Revista Caribeña de Ciencias Sociales. Recuperado de: https:/www.eumed.net/rev/caribe/2019/12/ intervencion-trabajador-social.html

Álava, Leila María y Veliz, Mónica del Rocío (2018). La intervención profesional del trabajador social frente a la violencia intrafamiliar. Revista Caribeña de Ciencias Sociales. Recuperado de: www.eumed.net/rev/caribe/2018/10/trabajador-social-violencia.html

Agrela, Belén; Gutiérrez, Camino y Fernández, Teresa (2017). Repensar la ética en Trabajo Social desde una perspectiva de género. Cuadernos de trabajo social, 30(1), 51-64. Recuperado de: https://core.ac.uk/download/pdf/81229701.pdf

Agrela, Belén y Morales, Amalia (2018). Trabajo social y estudios de género. Vindicando un espacio científico propio. Revista Estudo Feministas, 26(2), 2018.

Alcázar, Ana (2012). La Intervención Social en Centros de Acogida para Mujeres Víctimas de Violencia de Género en Andalucía. Análisis desde una Perspectiva de Género, 96-118. Recuperado de: https://factra.ujaen.es/sites/centro factra/files/ uploads/Congreso/6.pdf

Amoroso, Ivana y Fernández, Guadalupe (2014). Violencia de género y sistema de salud. Un análisis de la intervención hospitalaria. Limitaciones en la detección, reconocimiento afectivo y el abordaje desde el segundo nivel de atención. Debate público. Reflexión de Trabajo Social, 8, 91-100. Recuperado de: http://trabajosocial. sociales.uba.ar/wp-content/uploads/sites/13/2016/03/12 Fernandez.pdf

Aveiga, Vicenta y Ponce, José (2019). Una problemática de intervención del trabajador social: acoso sexual laboral. Mikarimin. Revista Científica Multidisciplinaria, 5(2). 117-126. Recuperado de: http://45.238.216.13/ojs/index.php/mikarimin/ article/view/1584

Bosch, Esperanza; Ferrer, Victoria y Alzamora, Aina (2005). Algunas claves para una psicoterapia de orientación feminista en mujeres que han padecido violencia de género. Feminismo/s, 6, 121-136. DOI: https: //doi.org/10.14198/fem.2005.6.09

Bravo, Cristina (2008). Menores víctimas de Violencia de Género: experiencia de intervención en un centro de acogida para familias víctimas de violencia de género. Psychosocial Intervention, 17(3), 337-351. Recuperado de http://scielo.isciii.es/ scielo.php? script=sci arttext\&pid=S1132-05592008000300009\&lng=es\&tlng=es.

Campos, Miriam, Commisso, Ángeles, Núñez, Mabel y Díaz, Rosario (2018). Trabajo Social y perspectiva de género. La primera entrevista a mujeres en situaciones 
de violencia. Ts. Territorios- Revista de trabajo social, (2), 23-46. Recuperado de: https://publicaciones.unpaz.edu.ar/OJS/index.php/ts/article/view/39/36

Cánovas, Rocío; Gallego, Beatriz; Navarro, María José y González, Víctor (2017). Violencia de género e intervención del trabajador social. ReiDoCrea, (8), 64-73. Recuperado de: https://www.ugr.es/ reidocrea/6-3-8.pdf

Castro-Serrano, Borja y Gutiérrez Olivares, Claudia. (2017). Intervención social y alteridad: una aproximación filosófica desde Lévinas. Andamios, 14(33), 217 239. Recuperado de: http://www.scielo.org.mx/scielo.php?script=sci arttext\&pi$\mathrm{d}=$ S1870-00632017000100217\&lng=es\&tlng=es.

Carrasco, Liliana y Martínez, Matías José (2020). Riesgos inminentes, cuerpos descorporizados, silencios que gritan, luchas colectivas o muerte. Efectos de la pandemia COVID-19 en la configuración de las violencias contra las mujeres. Red Sociales, Revista del Departamento de Ciencias Sociales, 7(2) 46-57.

Chacón, Laura Sofía (2019). La perspectiva de género como apuesta política en la intervención social de Trabajo Social. Prospectiva. Revista de Trabajo Social e intervención social, (28), 69-91. Recuperado de: https://doi.org/10.25100/prts. $\underline{\mathrm{v} 0 \mathrm{i} 28.8033}$

Clariá, Sofía; Condori, Sandra, y Rivolta, Yanina (2018). Trabajo Social y Violencia de Género: reflexiones en torno al ejercicio profesional en dispositivos públicos de acompañamiento a mujeres que atraviesan situaciones de violencia de género. Ts. Territorios-revista de trabajo social, (2), 9-21. Recuperado de: https://publicaciones. unpaz.edu.ar/OJS/index.php/ts/article/view/38

Duarte, Cory (2013). Procesos de construcción del Trabajo Social en Chile. De historia, feminización, feminismos y ciencias. Revista Eleuthera, (8), 253-270.

Duarte, Cory (2021). Despatriarcalizar el trabajo social: Propuesta para un trabajo social feminista e indisciplinado (en prensa).

Duarte, Cory y Rodríguez, Viviana (2020). Presencias y ausencias sobre género y feminismos en publicaciones de Trabajo Social. Perspectivas, 36, 253-285.

Elboj, Carmen y Ruíz, Laura (2010). Trabajo Social y Prevención de la Violencia de Género. Trabajo Social Global. Revista de Investigaciones en Intervención Social. 1(2), 220-233. Recuperado de: https://dialnet.unirioja.es/servlet/articulo?codigo $=5304681$

Falla, Uva (2017). La intencionalidad de la intervención del Trabajo Social. Trabajo Social hoy, 19, 123-135.

Roldán, Yanina; Failla, Sebastián y Hermida, María Eugenia (2019). Géneros, disidencias sexuales y decolonialidad en Ciencias Sociales. De problemas sociales a perspectivas críticas. II Jornadas de Sociología/UNMDP. Recuperado de: https:// fh.mdp.edu.ar/encuentros/index.php/jsoc/jsoc2019/paper/viewFile/5232/1352

Fernández, Patricia (2015). Trabajo Social Feminista: una revisión teórica para la redefinición práctica. Trabajo Social Global. Revista de Investigaciones en Intervención Social, 9(5), 24-39. Recuperado de: https://digibug.ugr.es/handle/10481/39329

Ford-Gilboe, Marilyn; Varcoe, Colleen; Scott-Storey, Kelly; Perrin, Nancy; Wuest, Judith; Wathen, Nadine; Case, James y Glass, Nancy (2020). Longitudinal impacts of an online safety and health intervention for women experiencing intimate partner violence: randomized controlled trial. BMC public health, 20(1), 1-17. Recuperado de: https://bmcpublichealth.biomedcentral.com/articles/10.1186/s12889-020$\underline{8152-8}$ 
Galaz, Caterine y Guarderaz, Paz (2016). La intervención psicosocial y la construcción de las “mujeres víctimas”. Una aproximación desde las experiencias de Quito (Ecuador) y Santiago (Chile). Revista de Estudios Sociales, 59, 68-82 Recuperado de: https://www.redalyc.org/pdf/815/81549422007.pdf

Guix, Joan (2007). El análisis de contenidos: ¿qué nos están diciendo?. Revista Calidad Asistencial, 1, 26-30. Recuperado de: https://www.elsevier.es/es-revista-revista-calidad-asistencial-256-pdf-S1134282X08704640

Hermida, María Eugenia (2017). "El Estado, el poder y la política en los estudios poscolonialesy el enfoque descolonial. Aportes para el Trabajo Social”. En P. Meschini, y M. E. Hermida (Eds.) (2017). Trabajo Social y Descolonialidad. Epistemologías insurgentes para la intervención en lo social. Mar del Plata, Argentina, Editorial de la Universidad Nacional de Mar del Plata.

Izquierdo, Rosana (2020). Violencia de género en tiempos del Covid-19. Cuadernos de atención primaria, 26(2), 26-27. Recuperado de: https://revista.agamfec. com/wp-content/uploads/2020/07/Agamfec-26 2-Violencia-de-g\%C3\%A9nero-en-tiempos-del-covid-19.pdf

Londoño, Nicolás (2020). Expresiones de la violencia basada en género, en el marco del confinamiento por COVID-19. Nova, 18 (35), 107-113. Recuperado de: https://doi.org/10.22490/24629448.4194

Lorente, Belén (2002). “Trabajo Social, mujer y perspectiva de género. Anotaciones para pensar la intervención social más allá de una intervención sectorial”. En M. Olza Zubiri y J. Hernández Arista (Ed.) Trabajo Social: (cuestiones sobre el qué y el cómo) (161-173). Zaragoza, España, Certez.

Lorente, Belén (2003). Perspectivas de género y trabajo social. Construyendo método desde el paradigma intercultural. Portularia, 3, 33-47. Recuperado de: http:// rabida.uhu.es/dspace/bitstream/handle/10272/224/b15131014.pdf?sequence

Lorente, Miguel (2021). Sociedad, COVID-19 y violencia de género (Society, COVID-19 and gender-based violence). Revista Española De Medicina Legal, Advance online publication. DOI: https://doi.org/10.1016/j.reml.2021.04.001

Lorente, Miguel (2020). Violencia de género en tiempos de pandemia y confinamiento. Revista Española de Medicina Legal, 46 (3), 139-145. DOI: 10.1016/j. reml.2020.05.005

Méndez, Concha y Álvarez-Buylla, Susana (2007). Reflexión crítica sobre la intervención con mujeres víctimas de violencia de género. Cuadernos de Trabajo Social, 20, 289-296. Recuperado de: https://revistas.ucm.es/index.php/CUTS/article/ view/CUTS0707110289A

Montenegro, Erica Marisel y Montenegro, Mónica Elisabet (2020). Re-pensando el campo de intervención del trabajo social junto a las víctimas de violencia de género, durante la pandemia de covid-19. Difusiones, 18(18), 59-67 Recuperado de: https:// revistadifusiones.net/index.php/difusiones/article/view/223

Muñoz, Gianinna (2014). Intervención social y la construcción epistemológica de la ciudadanía en Chile. Trabajo Social Global. Revista de Investigaciones en Intervención Social, 4(7), 36-57.

Muñoz, Gianinna (2018). The neoliberal turn in Chilean social work: frontline struggles against individualism and fragmentation. European Journal of Social Work, 1-12. DOI:10.1080/13691457.2018.1529657 
Muñoz, Gianinna; Reininger, Taly; Villalobos, Cristóbal; Morales, Carla (2021). "Análisis de las condiciones laborales de trabajadoras/es sociales que implementan programas en Chile en tiempos de COVID-19”. En S. Iturrieta (Ed.) Vivir en tiempos convulsionados Reflexiones sociocríticas para propuestas de intervención social, 10(2).

Nogueiras, Belén; Blanc, Pilar; Pliego, Purificación (2005). Los talleres y grupos de reflexión entre mujeres como prevención de la violencia. Una experiencia en una Concejalía de Mujer. Cuadernos De Trabajo Social, 18, 351-360. Recuperado de: https://revistas.ucm.es/index.php/CUTS/article/view/CUTS0505110351A

Orjuela, Astrid (2012). El concepto de violencia de género en el Derecho Internacional de los Derechos Humanos. Revista Latinoamericana de Derechos Humanos, 23(1), 89-114.

Penyarroja, Aina (2021). La influencia de las intervenciones profesionales en el proceso de salida de la violencia de género. Disjuntiva, 2(2), 34-45. DOI://doi. org/10.14198/DISJUNTIVA2021.2.2.3

Piedra, Julio, Rosa; Juan José, y Muñoz, María Concepción (2018). Intervención y prevención de la violencia de género: Un acercamiento desde el trabajo social. Trabajo social Global-Global Social Work, 8(14), 195-216. Recuperado de: https:// dialnet.unirioja.es/servlet/articulo? codigo $=6486589$

Plaza, Montserrat y Cantera, Leonor (2016). El impacto de la violencia de género en la maternidad: entrevistas en profundidad para reflexionar sobre las consecuencias y la intervención. Journal of Feminist, Gender and Women Studies, 1, 85-96. Recuperado de: https://revistas.uam.es/revIUEM/article/view/414/386

Quispe, Carlos (2020). Trabajo Social y el COVID-19: Perspectiva Política. Praxis Profesional y Posibilidad Comunitaria. Revista Margen. Recuperado de: https:// www.margen.org/pandemia/quispe.html

Ríos, Pilar (2003). Intervención desde el Trabajo Social en Situaciones de Malos Tratos Contra las Mujeres". Portularia, 3, 79-86. Recuperado de: http://rabida.uhu. es/dspace/bitstream/handle/10272/214/b15130642.pdf? sequence $=1$

Ríos, Pilar (2010). Violencia de Género y Pensamiento Profesional: Una Investigación sobre la Práctica del Trabajo Social. Trabajo Social Global. Revista de Investigaciones en Intervención Social, 1(1), 131-148.

Ríos, Pilar (2014). Intervención del trabajo social con mujeres maltratadas (Tesis doctoral). Universidad de Granada. Recuperado de: http://hera.ugr.es/tesisugr/21599245.pdf

Romero, Janet (2019). Violencia de género y Trabajo Social. Análisis de los discursos y abordajes de los/las profesionales de Trabajo Social en materia de violencia de género. Revista de Treball Social 217, 31-53. Recuperado de: https://www.revistarts.com/es/node/910

Ruiz, Isabel y Pastor, Guadalupe (2021). Medidas de contención de la violencia de género durante la pandemia de COVID-19. Gaceta Sanitaria, 35(4), 389-394. Recuperado de: https://doi.org/10.1016/i.gaceta.2020.04.005.

Sánchez, Agustina y Percara, Camila (2017). Aportes para pensar la intervención en situaciones de violencia de género a partir de una experiencia de prácticas. Voces emergentes, 1(1), 10-15. Recuperado de: http://sedici.unlp.edu.ar/hand$\underline{\text { le/10915/63750 }}$ 
Santana, Juana (2010). La formación para el Trabajo Social en contextos de desigualdad de género y violencia contra las mujeres, Portularia 10(2), 91-99. Recuperado de: http://rabida.uhu.es/dspace/bitstream/handle/10272/4312/b15971016. pdf? sequence $=2$

Santana, Juana y Gonzales, Rosaura (2013). Una mirada a la experiencia vivida por mujeres que han roto una relación de pareja violenta. Portularia, 11(2), 49- 57. Recuperado de: https://www.redalyc.org/pdf/1610/161028683005.pdf

Strier, Roni y Bershtling, Orit (2016). Professional Resistance in Social Work: Counterpractice Assemblages. Social Work, 61(2), 111-118. DOI:10.1093/sw/sww010

Tudela, María Pilar (2015). Voces migrantes frente a la violencia de género: una investigación para la acción en San Francisco (EE. UU). Cuadernos de Trabajo Social, 22, 185-206. Recuperado de: https://doi.org/10.14198/ALTERN2015.22.11

Ullastre, Jimena (2018). Violencia de género: una experiencia de intervención desde un efector del primer nivel de atención de la salud pública. PLAZA PÚBLICA. Revista de Trabajo Social, (20), 62-73. Recuperado de: http://ojs2.fch.unicen.edu. ar:8080/ojs-3.1.0/index.php/plaza-publica/article/view/334

Villaluenga, Maite (2020). Violencia de género y salud: perspectiva desde el Trabajo Social Sanitario. Almoraima. Revista de Estudios Campogibraltareños, 44, 8189. Recuperado de: http://institutoecg.es/wp-content/uploads/2020/06/Maite-Villaluenga.pdf

Zamanillo, Teresa (2004) Hermenéutica de la intervención social. En E. Roldán (Ed.) Género, políticas sociales e intervención social (70-117). Madrid, España, Editorial Complutense.

Recibido: 31/07/2021

Aceptado: 05/10/2021 